\title{
Dietary interventions for adult cancer survivors (Protocol)
}

\author{
Burden S, Gibson DJ, Todd C, Gratton EK, Pilling M, Lal S
}

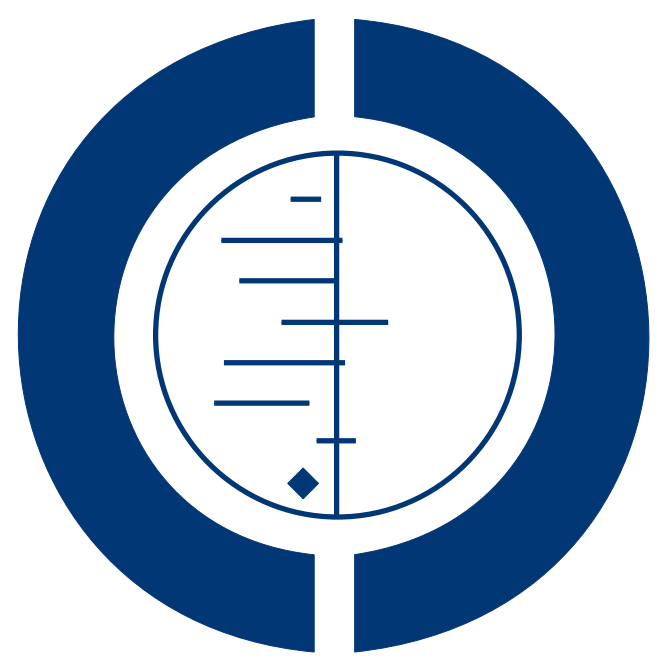

\section{THE COCHRANE COLLABORATION $^{\circledR}$}

This is a reprint of a Cochrane protocol, prepared and maintained by The Cochrane Collaboration and published in The Cochrane Library 2014, Issue 9

http://www.thecochranelibrary.com

\section{WILEY}


TABLE OF CONTENTS

HEADER . . . . . . . . . . . . . . . . . . . . . . . . . . . . . . . . . . . . 1

ABSTRACT . . . . . . . . . . . . . . . . . . . . . . . . . . . . . . . . . . . . . . . . . . . . .

BACKGROUND . . . . . . . . . . . . . . . . . . . . . . . . . . . . . . . . . . . .

OBJECTIVES . . . . . . . . . . . . . . . . . . . . . . . . . . . . . . . . . . . . . . . . . .

METHODS . . . . . . . . . . . . . . . . . . . . . . . . . . . . . . . . . . . . . .

ACKNOWLEDGEMENTS . . . . . . . . . . . . . . . . . . . . . . . . . . . . . . . . . . . . . . .

REFERENCES . . . . . . . . . . . . . . . . . . . . . . . . . . . . . . . . . . . . . . 5

APPENDICES . . . . . . . . . . . . . . . . . . . . . . . . . . . . . . . . . . . . . 7

CONTRIBUTIONS OF AUTHORS . . . . . . . . . . . . . . . . . . . . . . . . . . . . . . . . . . . . . . . . . .

DECLARATIONS OF INTEREST . . . . . . . . . . . . . . . . . . . . . . . . . . . . . . . . . . . . 8

SOURCES OF SUPPORT . . . . . . . . . . . . . . . . . . . . . . . . . . . . . . . . . . .

Dietary interventions for adult cancer survivors (Protocol)

Copyright @ 2014 The Cochrane Collaboration. Published by John Wiley \& Sons, Ltd. 


\title{
[Intervention Protocol]
}

\section{Dietary interventions for adult cancer survivors}

\author{
Sorrel Burden ${ }^{1}$, Debra J Gibson ${ }^{1}$, Chris Todd $^{1}$, Eleanor K Gratton ${ }^{2}$, Mark Pilling ${ }^{1}$, Simon Lal ${ }^{3}$ \\ ${ }^{1}$ School of Nursing, Midwifery and Social Work, University of Manchester, Manchester, UK. ${ }^{2}$ Nutrition and Dietetics, City Hospitals \\ Sunderland NHS Foundation Trust, Sunderland, UK. ${ }^{3}$ Intestinal Failure Unit, Salford Royal Foundation Trust, Salford, UK \\ Contact address: Sorrel Burden, School of Nursing, Midwifery and Social Work, University of Manchester, Room 6.32, Jean McFarlane \\ Building, Oxford Road, Manchester, M13 9PL, UK. Sorrel.burden@manchester.ac.uk.
}

Editorial group: Cochrane Gynaecological Cancer Group.

Publication status and date: New, published in Issue 9, 2014.

Citation: Burden S, Gibson DJ, Todd C, Gratton EK, Pilling M, Lal S. Dietary interventions for adult cancer survivors. Cochrane Database of Systematic Reviews 2014, Issue 9. Art. No.: CD011287. DOI: 10.1002/14651858.CD011287.

Copyright (C) 2014 The Cochrane Collaboration. Published by John Wiley \& Sons, Ltd.

\begin{abstract}
A B S T R A C T
This is the protocol for a review and there is no abstract. The objectives are as follows:

To assess the effects of dietary interventions for adult cancer survivors on morbidity and mortality, changes in dietary behaviour, body composition, health-related quality of life and clinical measurements.
\end{abstract}

\section{B A C K G ROU N D}

There are reported to be more than 24 million cancer survivors worldwide, based on data derived from population-based cancer registers (Parkin 2006). This includes 10 million survivors of cancer in the United States (Ganz 2005) and 2 million in the United Kingdom, along with an estimated 3\% increase annually (Maddams 2009). The proportion of people who survive cancer is increasing, which may be attributed to an increase in the aging population and advancements in anti-cancer therapies (chemotherapy and radiotherapy), which have improved the outcomes of treatments (Aziz 2003; Lancet 2004). Over 60\% of those living beyond a cancer diagnosis are over 65 years of age (Ravasco 2003), approximately $60 \%$ are female, and the majority are diagnosed initially with either breast, prostate or colorectal malignancy (Maddams 2009). However, negative factors influencing cancer survival have been highlighted and include people who have a lower socioeconomic status combined with higher levels of co-existing conditions and unhealthy lifestyle choices (Louwman 2010). It is now recognised that as survival increases, associated long-term health issues of cancer will emerge as a significant public health concern (Mosher 2009) and this is reflected in healthcare strategies (Department 2010; Lippman 2004).

Health promotion initiatives aimed at improving the well-being of people who have survived cancer are now essential in order to decrease co-morbidities and improve quality of life (QoL). Focus groups have reported that people who have survived cancer are often confused regarding future strategies to improve their health and well-being (Armes 2009; Marbach 2011).

\section{Description of the condition}

For the purpose of this review, cancer survivors are defined as people living beyond a diagnosis of cancer after all treatment interventions have been discontinued, where treatment interventions may include surgery, chemotherapy, radiotherapy and active hormone replacement therapy. Those receiving adjuvant maintenance hormone treatments will be included. This review does not include the patients with cancer who are currently undergoing active or palliative treatment.

Dietary interventions for adult cancer survivors (Protocol)

Copyright $\odot 2014$ The Cochrane Collaboration. Published by John Wiley \& Sons, Ltd. 


\section{Description of the intervention}

International recommendations on how to maintain healthy lifestyles are currently available for the prevention of cancer and also for those who are living beyond a cancer diagnosis from the World Cancer Research Fund and American Institute for Cancer Research (WCRF/AICR 2007; Kushi 2012). Healthy lifestyle changes recommended by the WCRF/AICR 2007 have been linked to longevity. From a large European study, those who followed a higher proportion of healthy lifestyle recommendations had a $34 \%$ lower hazard ratio (HR) of death compared to those who adhered to fewer recommendations (Vergnaud 2013). Low compliance with the WCRF/AICR recommendations was significantly associated with increased HRs of dying from cancer, circulatory and respiratory disease (Vergnaud 2013). The healthy lifestyle recommendations for those living beyond cancer include: maintaining a healthy weight throughout life; adopting an active lifestyle; consuming a healthy diet with an emphasis on plant foods; and limiting alcoholic beverages (WCRF/AICR 2007). Dietary interventions include any method of delivering an intervention that is aimed at altering an individual's food or drink intake.

\section{How the intervention might work}

Lifestyle factors predispose people to the development of chronic diseases and cancer. These include being overweight or obese, lack of physical activity and high saturated fat intake combined with a low intake of fruit and vegetables (Daar 2007). There is a plethora of data linking chronic diseases, including diabetes, cardiovascular, and respiratory diseases, to lifestyle factors, so it would seem reasonable that these co-morbidities, in people who have survived cancer, could be reduced by modifying lifestyle factors (Kushi 2012). Those who live beyond cancer also have an elevated incidence of recurrent disease and other cancers, so would potentially benefit from modifying their behaviour to adhere to the recommendations for cancer prevention. Furthermore, patients have been found to have a higher level of motivation to change lifestyle behaviours after a cancer diagnosis than prior to their diagnosis (Demark-Wahnefried 2005; Ganz 2005; Satia 2004). A survey of modifications in health-related behaviours demonstrated that two thirds of people surviving breast, colorectal and prostate cancer made positive health-related changes to their diet and changed supplement usage up to two years after their cancer diagnosis (Patterson 2003). Others have concurred that patients are willing to change their behaviour after a diagnosis of cancer and have already made changes (Demark-Wahnefried 2000).

\section{Why it is important to do this review}

Those who have survived cancer not only have an increased risk of secondary malignancies, but also a higher incidence of comorbidities, compared to the general population (Nord 2005).
An increased incidence of cardiovascular disease, diabetes and osteoporosis have been reported in survivors of cancer (DemarkWahnefried 2009; Hawkes 2011; Janssen-Heijnen 2009). Genotype and lifestyle are also considered significant contributory factors that lead to increased morbidity and cancer recurrence in people who have survived cancer (Daar 2007; Demark-Wahnefried 2009). Furthermore, survivors of cancer use healthcare services and receive social welfare benefits more frequently than controls (Nord 2005). In addition, it has been shown that those who have survived cancer visit their general practitioners more frequently than their non-cancer counterparts (Khan 2011). Research has demonstrated that the poorer health status identified in survivors of cancer detrimentally influences QoL (Baker 2003). In older people who have survived cancer, improved diet and enhanced physical activity has been shown to relate to better vitality and functioning (Hewitt 2003).

This systematic review is important to determine which dietary interventions are effective in those who have survived cancer. There is now evidence to support exercise initiatives in cancer survivors, in relation to health-related QoL (Mishra 2012). In the promotion of lifestyle behaviours it is difficult to unravel the contribution of individual components on overall health and well-being. However, it would be useful to determine the most appropriate dietary interventions that are effective in people who have survived cancer, to inform clinical practitioners, and also to assist in improving the long-term health of people who have survived cancer. Evidence on dietary interventions in survivors of cancer is now developing, so it is timely to review the literature to summarise the research, in order to inform clinical practice and policy development, and identify gaps in the literature for further research.

\section{O B J E C T I VES}

To assess the effects of dietary interventions for adult cancer survivors on morbidity and mortality, changes in dietary behaviour, body composition, health-related quality of life and clinical measurements.

\section{METHOD S}

\section{Criteria for considering studies for this review}

\section{Types of studies}

We will include randomised controlled trials (RCTs) and cluster RCTs published in peer reviewed journals. We will also contact investigators of eligible unpublished studies identified from the 
abstracts of conference proceedings for relevant unpublished data and search trial registries for additional studies.

\section{Types of participants}

All adult cancer survivors, defined as those who have lived beyond a cancer diagnosis which occurred after the age of 18 years, and have completed all active anti-cancer interventions, such as surgery, radiotherapy, chemotherapy or active hormone replacement (patients receiving adjuvant maintenance hormone replacement will be included). Patients with pre-cancerous lesions will not be included.

\section{Types of interventions}

All dietary interventions for healthy eating and weight loss or weight maintenance. Specific nutritional interventions, including only those based on food, will be included. Dietary interventions need to include multiple nutrients, fat, carbohydrate, protein, vitamins and minerals. Oral supplements, including those with single or multiple nutrients, are to be excluded. Probiotic supplements will be excluded, along with all intravenous nutrient solutions containing single or multiple nutrient administrations. All enteral feeding will also be excluded.

\section{Types of outcome measures}

\author{
Primary outcomes \\ - Overall survival \\ - Incidence of secondary malignancy or other cancer \\ - Incidence of co-morbidities
}

\section{Secondary outcomes}

- Dietary changes measured by dietary analysis using food frequency questionnaires, dietary recall, food diaries or assessed by dietary assessment methodology

- Changes in body weight or anthropometric measurements including hip and waist ratios, skin fold thickness, or functional capacity measurements

- Patient - outcomes, including quality of life (QoL) questionnaires

- Biochemical measurements, which may include lipid profiles or serum glucose as surrogate markers

- Number of healthy eating changes made to habitual eating patterns

\section{Search methods for identification of studies}

\section{Electronic searches}

We will search the following electronic databases:

- the Cochrane Central Register of Controlled Trials

(CENTRAL, The Cochrane Library, latest issue)

- the Cochrane Gynaecological Cancer Review Group's Trial

Register

- MEDLINE (Ovid) (1946 to latest issue)

- EMBASE (Ovid) (1980 to latest issue)

- AMED

- CINAHL

- DARE

We present the MEDLINE search strategy in Appendix 1. For databases other than MEDLINE, the search strategy will be adapted accordingly. All relevant articles will be identified on PubMed and using the 'related articles' feature, and a further search will be carried out for newly published articles. Reports in all languages will be sought and translations carried out if necessary.

\section{Searching other resources}

We will review the reference list of all retrieved articles and other reviews on the topic. We will also search World Health Organization (WHO) International Clinical Trials Registry Platform ( www.who.int/ictrp/en).

We will search MetaRegister (www.controlled-trials.com), Physicians Data query (nci.nih.gov), www.clinicaltrials.gov and www.cancer.gov/clinicaltrials for ongoing trials. If ongoing trials that have not been published are identified through these searches, the principal investigators will be approached to ask for relevant data. Conference proceedings and abstracts will be searched through ZETOC (zetoc.mimas.ac.uk) and WorldCat Dissertations.

We will handsearch the abstracts from meetings held by the American Institute for Cancer Research.

\section{Data collection and analysis}

\section{Selection of studies}

Two review authors (DG and EG) will independently assess the titles and abstracts retrieved from the searches to determine relevance and eligibility. All papers failing to meet the eligibility criteria will be excluded. Full text articles of potentially relevant studies will be retrieved and reviewed by two review authors independently, to assess whether they meet the inclusion criteria. We will record the selection process in sufficient detail to complete a PRISMA flow diagram and characteristics of included or excluded

Dietary interventions for adult cancer survivors (Protocol)

Copyright $\odot 2014$ The Cochrane Collaboration. Published by John Wiley \& Sons, Ltd. 
studies tables. A third review author will be called upon to resolve any conflicts that arise during study selection. Multiple reports of the same study will be linked. We will translate any non-English articles before assessment, as required.

\section{Data extraction and management}

A standardised data collection form will be devised to facilitate data collection from the included studies. The data extraction form will be piloted and modified as required. Two review authors (DG and EG) will independently extract data and any discrepancies will be discussed with a third author. The following information will be recorded for each trial:

- Year of publication, country of origin, source of funding and number of participants.

- Study population

- Age, gender, location of tumour, previous therapy, cancer staging or classification.

- Other baseline characteristics, including proportion of overweight or obese survivors (defined by body mass index greater than $25 \mathrm{~kg} / \mathrm{m}^{2}$ or nutrition status assessment derived from a validated tool), alcohol intake, smoking status, current physical activity and socioeconomic group.

- Overall survival

- Incidence of secondary malignancy or other cancer

- Incidence of co-morbidities

- Dietary changes measured by dietary analysis using food frequency questionnaires, dietary recall, food diaries or assessed by dietary assessment methodology

- Changes in body weight or anthropometric measurements including hip and waist ratios, skin fold thickness, or functional capacity measurements

- Patient outcomes, including quality of life (QoL)

questionnaires

- Biochemical measurements, which may include lipid profiles or serum glucose as surrogate markers.

- Number of healthy eating changes made to habitual eating patterns

- Details of type of intervention, including nutritional education, change behaviour techniques employed and delivery method of intervention (written, telephone, face to face or Internet based).

\section{Assessment of risk of bias in included studies}

The risk of bias in included studies will be assessed using the Cochrane Collaboration's tool (Higgins 2011). This will include assessment of the following:

- Selection bias

$\circ$ random sequence generation

- allocation concealment

- Performance bias
- blinding of participants and personnel (patients and treatment providers), although this may not be possible due to the nature of some of the interventions

- Detection bias

$\circ$ blinding of outcome assessment

- Attrition bias

$\circ$ incomplete outcome data: We will record the proportion of participants whose outcomes were not reported at the end of the study and categorise as follows;

$\diamond$ low risk of bias, if fewer than $20 \%$ of patients were lost to follow up and reasons for loss to follow up were similar in both treatment arms

$\diamond$ high risk of bias, if more than $20 \%$ of patients lost to follow up or reasons for loss to follow up differed between treatment arms

$\diamond$ unclear risk of bias if loss to follow up was not

reported

- Reporting bias

- selective reporting of outcomes

- Other possible sources of bias

Two review authors will independently apply the 'Risk of bias' tool and differences will be resolved by discussion or by appeal to a third review author. Results will be summarised in both a 'Risk of bias' graph and a 'Risk of bias' summary. Results of meta-analyses will be interpreted in light of the findings with respect to risk of bias.

\section{Measures of treatment effect}

The measurement of treatment effect will be expressed as follows. For dichotomous variables, risk ratios (RR) will be calculated and expressed with $95 \%$ confidence intervals (CI). For continuous data expressed as means with standard deviations, we will use mean difference to show effect size. For data presented as time-to-event, if they are dichotomous, odds ratios will be used and for a log rank approach we will calculate hazard ratios (HR).

\section{Unit of analysis issues}

We will include cluster randomised trials. In these trials individuals are randomised as a block, from one centre or one clinic, so this will be dealt with on a trial by trial basis, depending on the study design.

\section{Dealing with missing data}

An intention-to-treat analysis is planned and we will contact authors for any missing 'Risk of bias' information or outcome data required, if appropriate. We will report on the levels of loss to follow-up and assess this as a source of potential bias. We will investigate, through sensitivity analyses, the effects of any imputed data on pooled effect estimates.

Dietary interventions for adult cancer survivors (Protocol)

Copyright $\odot 2014$ The Cochrane Collaboration. Published by John Wiley \& Sons, Ltd. 


\section{Assessment of heterogeneity}

We will assess the heterogeneity of any combined studies in the meta-analysis using $\mathrm{I}^{2}$. If $\mathrm{I}^{2}$ is greater than $30 \%$ we will examine possible reasons for heterogeneity in relation to clinical setting, study participants and similarity of clinical parameters in studies.

\section{Assessment of reporting biases}

We will search multiple sources including trial registries as detailed above. We will consider whether trials were undertaken and reported according to their trial protocol. If there is a sufficient number of included studies, we will attempt to assess publication bias using a funnel plot as detailed in section 10.4 of the Cochrane Handbook for Systematic Reviews of Interventions (Higgins 2011).

\section{Data synthesis}

Review Manager 2014 will be used for any data synthesis. Metaanalyses will only be conducted if there are studies reporting similar comparisons for the same outcomes. Meta-analyses will be performed using the Mantel-Haenszel random-effects method for the synthesis of the dichotomous data, due to the anticipated level of heterogeneity in the studies.

For continuous variables, inverse variance will be used in a random-effects method if there are suitable data to perform a metaanalysis. If there are enough studies reporting time-to-event data then Peto method will be used in a fixed-effect model if a metaanalysis is appropriate (random-effects model is not available for Peto method in Review Manager 2014). If we establish that there is significant heterogeneity ( $\mathrm{I}^{2}$ more than $30 \%$ ) between the studies, we will not undertake a meta-analysis but will investigate possible causes of heterogeneity. If a meta-analysis cannot be undertaken we will undertake a descriptive review of the studies.

\section{Subgroup analysis and investigation of heterogeneity}

If the data allow, planned subgroup analysis will be undertaken on different cancer types and also on different dietary intervention methods delivered for specific interventions. This could include subgroup analysis of interventions for weight management, or analysis which looks at interventions delivered by telephone interviewing.

\section{Sensitivity analysis}

Sensitivity analysis will be undertaken to evaluate the effects of bias on the results by investigating the impact of trials that have a high level of bias or an unclear level of bias. Each of the items assessed to indicate bias will bias evaluated separately.

\section{ACKNOW LEDGEMENTS}

The National Institute for Health Research (NIHR) is the largest single funder of the Cochrane Gynaecological Cancer Group. The views and opinions expressed therein are those of the authors and do not necessarily reflect those of the NIHR, NHS or the Department of Health.

\section{REF E R E NCES}

\section{Additional references}

\section{Armes 2009}

Armes J, Crowe M, Colbourne L, Morgan H, Murrells T, Oakley C, et al.Patients' supportive care needs beyond the end of cancer treatment: a prospective, longitudinal survey. Journal of Clinical Oncology 2009;27(36):6172-9. [DOI: 10.1200/JCO.2009.22.5151]

Aziz 2003

Aziz NM, Rowland JH. Trends and advances in cancer survivorship research: challenge and opportunity. Seminars in Radiation Oncology 2003;13(3):248-66.

\section{Baker 2003}

Baker F, Haffer SC, Denniston M. Health-related quality of life of cancer and noncancer patients in Medicare managed care. Cancer 2003;97(3):674-81.

Daar 2007

Daar AS, Singer PA, Persad DL, Pramming SK, Matthews $\mathrm{DR}$, Beaglehole R, et al.Grand challenges in chronic noncommunicable diseases. Nature 2007;7169:494-6.

\section{Demark-Wahnefried 2000}

Demark-Wahnefried W, Peterson B, McBride C, Lipkus I, Clipp E. Current health behaviours and readiness to pursue life-style changes among men and women diagnosed with early stage prostate and breast carcinomas. Cancer 2000;88 (3):674-84

\section{Demark-Wahnefried 2005}

Demark-Wahnefried W, Aziz NM, Rowland JH, Pinto BM Riding the crest of the teachable moment: promoting longterm health after the diagnosis of cancer. Journal of Clinical Oncology 2005;23(24):5814-30.

\section{Demark-Wahnefried 2009}

Demark-Wahnefried W, Jones LW. Promoting a healthy lifestyle among cancer survivors. Hematology - Oncology Clinics of North America 2009;22(2):319-42.

Department 2010

Department of Health Macmillan Cancer Support \& NHS Improvements. National Cancer Survivorship Initiative: Vision. www.dh.gov.uk/publications 2010; Vol. 299671/ National Cancer Survivorship Initiative - Vision. 
Ganz 2005

Ganz PA. A teachable moment for oncologists: cancer survivors, 10 million strong and growing!. Journal of Clinical Oncology 2005;23(24):5458-60.

\section{Hawkes 2011}

Hawkes AL, Lynch BM, Owen N, Aitken JF. Lifestyle factors associated concurrently and prospectively with comorbid cardiovascular disease in a population-based cohort of colorectal cancer survivors. European Journal of Cancer 2011;47(2):267-76.

\section{Hewitt 2003}

Hewitt M, Rowland JH, Yancik R. Cancer survivors in the United States: age, health, and disability. Journals of Gerontology. Series A, Biological Sciences and Medical Sciences 2003;58(1):M82-91.

\section{Higgins 2011}

Higgins JPT, Green S (editors). Cochrane Handbook for Systematic Reviews of Interventions Version 5.1.0 [updated March 2011].. The Cochrane Collaboration Availablefrom www.cochrane-handbook.org. The Cochrane Collaboration

\section{Janssen-Heijnen 2009}

Janssen-Heijnen MLG, Szerencsi K, van de Schans SAM, Maas Huub AAM, Widdershoven JW, Coebergh JWW. Cancer patients with cardiovascular disease have survival rates comparable to cancer patients within the age-cohort of 10 years older without cardiovascular morbidity. Critical Reviews in Oncology/Hematology 2009;76(3):196-207.

\section{Khan 2011}

Khan NF, Ward AM, Watson E, Rose PW. Consulting and prescribing behaviour for anxiety and depression in longterm survivors of cancer in the UK. European Journal of Cancer 2011;46(18):3339-44.

\section{Kushi 2012}

Kushi LH, Doyle C, McCullough M, Rock CR, DemarkWahnefried W, Bandera EV, et al and The American Cancer Society 2010 Nutrition and Physical Activity Guidelines Advisory Committee. American Cancer Society Guidelines on Nutrition and Physical Activity for Cancer Prevention. Reducing the risk of cancer with healthy food choices and physical activity. CA: A Cancer Journal for Clinicians 2012; 62:30-67.

\section{Lancet 2004}

Editorial. Cancer survivors: living longer, and now, better. Lancet 2004;364(9452):2153-4.

\section{Lippman 2004}

Lippman SM, Levin B, Brenner DE, Gordon GB, Aldige $\mathrm{CR}$, Kramer BS, et al.Cancer prevention and the American Society of Clinical Oncology. Journal of Clincial Oncology 2004;22(19):3848-51.

\section{Louwman 2010}

Louwman WJ, Aarts MJ, Houterman S, van Lenthe FJ, Coebergh JWW, Janssen-Heijnen MLG. A 50\% higher prevalence of life-shortening chronic conditions among cancer patients with low socioeconomic status. British Journal of Cancer 2010;103(11):1742-8.

\section{Maddams 2009}

Maddams J, Brewster D, Gavin A, Steward J, Elliott J, Utley M, et al.Cancer prevalence in the United Kingdom: estimates for 2008. British Journal of Cancer 2009;101(3): 541-7.

\section{Marbach 2011}

Marbach TJ, Griffie J. Patient preferences concerning treatment plans, survivorship care plans, education, and support services. Oncology Nursing Forum 2011;38(3): $335-42$.

\section{Mishra 2012}

Mishra SI, Scherer RW, Geigle PM, Berlanstein DR, Topaloglu O, Gotay CC, et al.Exercise interventions on health-related quality of life for cancer survivors. Cochrane Database of Systematic Reviews 2012, Issue 8. [DOI: 10.1002/14651858.CD007566.pub2]

\section{Mosher 2009}

Mosher CE, Sloane R, Morey MC, Snyder DC, Cohen HJ, Miller PE, et al.Associations between lifestyle factors and quality of life among older long-term breast, prostate, and colorectal cancer survivors. Cancer 2009;115(17):4001-9.

\section{Nord 2005}

Nord C, Mykletun A, Thorsen L, Bjøro T, Fosså SD. Selfreported health and use of health care services in long-term cancer survivors. International Journal of Cancer 2005;114 (2):307-16

\section{Parkin 2006}

Parkin DM, Fernández LMG. Use of statistics to assess the global burden of breast cancer. Breast Journal 2006;12: S70-80.

\section{Patterson 2003}

Patterson RE, Neuhouser ML, Hedderson MM, Schwartz SM, Standish LJ, Bowen DJ. Changes in diet, physical activity, and supplement use among adults diagnosed with cancer. Journal of the American Dietetic Association 2003; 103(3):323-8.

\section{Ravasco 2003}

Ravasco P, Monteiro-Grillo I, Vidal PM, Camilo ME. Nutritional deterioration in cancer: the role of disease and diet. Clinical Oncology 2003;15(8):443-50.

\section{Review Manager 2014}

Copenhagen: The Nordic Cochrane Centre, The Cochrane Collaboration. Review Manager (RevMan). Version 5.3. Copenhagen: The Nordic Cochrane Centre, The Cochrane Collaboration, 2014.

\section{Satia 2004}

Satia JA, Campbell MK, Galanko JA, James A, Carr C, Sandler RS. Longitudinal changes in lifestyle behaviors and health status in colon cancer survivors. Cancer Epidemiology Biomarkers \& Prevention 2004;13(6):1022-31.

\section{Vergnaud 2013}

Vergnaud A-C, Romaguera D, Peeters, PH, van Gils CH, Chan DS, Romieu I, et al.Adherence to the World Cancer Research Fund/American Institute for Cancer Research guidelines and risk of death in Europe: results from the 
European Prospective Investigation into Nutrition and

Cancer cohort study. American Journal of Clinical Nutrition

2013;97(5):1107-20.

\section{WCRF/AICR 2007}

WCRF/AICR. Food, nutrition and the prevention of cancer.

A Global Perspective Expert Report. World Cancer Research

Fund, 2007.

* Indicates the major publication for the study

\section{A P P E N D I C E S}

\section{Appendix I. MEDLINE Ovid search strategy}

1 exp neoplasms/

2 (cancer* or tumor* or tumour* or neoplas* or malignan* or carcinoma* or adenocarcinoma* or choriocarcinoma* or leukemia* ${ }^{*}$ leukaemia* or metastat* or sarcoma* or teratoma*).ti,ab.

31 or 2

4 Diet/

5 exp nutrition assessment/

$6 \exp$ Nutrition Therapy/

7 exp Nutrition Disorders/

8 Food Habits/

9 Food Preferences/

10 exp Food/

11 (diet* $^{*}$ or nutrition* or nutrient* $^{*}$ or food* or feed $^{*}$ or eat* or drink*).ti,ab.

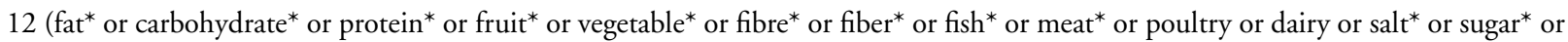
cereal* $^{*}$ or nut* ${ }^{*}$ or seed* or alcohol* or caffeine).ti,ab.

13 (macrobiotic or ketogenic or vegetarian or (low adj (glycemic* or glycaemic $\left.\left.{ }^{*}\right)\right)$ ).ti,ab.

144 or 5 or 6 or 7 or 8 or 9 or 10 or 11 or 12 or 13

15 survivors/

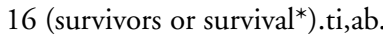

1715 or 16

183 and 14 and 17

19 randomized controlled trial.pt.

20 controlled clinical trial.pt.

21 randomized.ab.

22 placebo.ab.

23 clinical trials as topic.sh.

24 randomly.ab.

25 trial.ti.

2619 or 20 or 21 or 22 or 23 or 24 or 25

2718 and 26

28 exp animals/ not humans.sh.

2927 not 28

key:

[mp=title, abstract, original title, name of substance word, subject heading word, protocol supplementary concept, rare disease supplementary concept, unique identifier]

Dietary interventions for adult cancer survivors (Protocol)

Copyright $\odot 2014$ The Cochrane Collaboration. Published by John Wiley \& Sons, Ltd. 


\section{CONTRIBUTIONSOFAUTHORS}

SB wrote the proposal and SL, EG and DG have commented on the nutritional and clinical components of the protocol. The methodological aspects of the systematic review protocol have been commented on by CT and MP has reviewed the plan for data analysis.

The National Institute for Health Research (NIHR) is the largest single funder of the Cochrane Gynaecological Cancer Group. The views and opinions expressed herein are those of the authors and do not necessarily reflect those of the NIHR, National Health Service or Department of Health.

\section{DECLARATIONSOF INTEREST}

None known

\section{SOURCES OF SUPPORT}

\section{Internal sources}

- No sources of support supplied

\section{External sources}

- Macmillan Cancer Care, UK.

Dr Burden was supported by a Post Doctoral Fellowship 